\title{
THE IMPACT OF THE AVAILABILITY OF RESOURCES, THE ALLOCATION OF BUFFERS AND NUMBER OF WORKERS ON THE EFFECTIVENESS OF AN ASSEMBLY MANUFACTURING SYSTEM
}

\author{
Sławomir Kłos ${ }^{1}$, Peter Trebuna ${ }^{2}$ \\ ${ }^{1}$ University of Zielona Góra, Institute of Management and Production Engineering, Poland \\ 2 Technical University of Kosice, Faculty of Mechanical Engineering, Slovakia \\ Corresponding author: \\ Stawomir Klos \\ University of Zielona Góra \\ Institute of Management and Production Engineering \\ Prof. Szafrana 4, 65-526 Zielona Góra, Poland \\ phone: (+48) 68 3282464 \\ e-mail:s.klos@iizp.uz.zgora.pl
}

Received: 7 September 2016 Accepted: 31 May 2017

\begin{abstract}
This paper proposes the application of computer simulation methods in order to analyse the availability of resources, buffers and the impact of the allocation of workers on the throughput and work-in-progress of a manufacturing system. The simulation model of the production system is based on an existing example of a manufacturing company in the automotive industry. The manufacturing system includes both machining and assembly operations. Simulation experiments were conducted vis-à-vis the availability of the different manufacturing resources, the various allocations of buffer capacities and the number of employees. The production system consists of three manufacturing cells - each cell including two CNC machines - and two assembly stations. The parts produced by the manufacturing cells are stored in buffers and transferred to the assembly stations. Workers are allocated to the manufacturing cells and assembly stations, but the number of workers may be less than number of workplaces and are thus termed 'multi-workstations'. Using computer simulation methods, the impact of the availability of resources, the number of employees and of the allocation of buffer capacity on the throughput and work-in-progress of the manufacturing system is analysed. The results of the research are used to improve the effectiveness of manufacturing systems using a decision support system and the proper control of resources. Literature analysis shows that the study of the impact of buffer capacities, availability of resources and the number of employees on assembly manufacturing system performance have not been carried out so far.
\end{abstract}

KEYWORDS

computer simulation, availability of resources, buffer allocation, throughput analysis, workin-progress.

\section{Introduction}

The effectiveness of a production system is often a very important aspect in deciding the competiveness of manufacturing enterprises. The automotive sector, in particular, requires high throughput and quality from its parts suppliers. Relatively low profit margins limit the technological possibility for small and medium companies, which produce many hundreds of thousands of parts monthly, to increase their effectiveness. However, many manufacturing enterprises have huge potential to effectively improve their production planning and control by the implementation of new methods and re-tooling. Increases in throughput in a manufacturing system can be reached by the proper allocation of buffer capacities, employees and by improving the availability of resources.

Because of the variety of manufacturing system structures and the different assumptions and limitations concerned with manufacturing orders, it is very difficult to find optimal solution using mathematical models. Most of the combinatorial optimisation 
problems are NP-hard. Therefore, the implementation of computer simulation methods to analyse the behavior of individual systems enables interesting results to be obtained over a relatively short period of time. Of course simulation does not guarantee finding any optimal solution but on the basis of a relatively small number of experiments, sufficient solutions can be found.

Simulation is a very important research method used for the modelling, analysis and development of manufacturing systems. Many scientific papers include the application of computer simulation in the general design of manufacturing systems and in the analysis of the operational, production planning and scheduling of manufacturing systems [1]. Jahangirian et al., report the results of a review of the application of simulations, published within peer-reviewed literature, between 1997 and 2006 and the analysis of the role of simulation techniques within manufacturing and business [2]. Jagstam and Klingstam use discrete event simulation as an aid to conceptual design and the pre-study of manufacturing systems through developing a virtual factory. They propose a simulation handbook to fully integrate simulation as a tool in engineering processes. They also identify the problems associated with integration into the design of manufacturing systems of discrete event simulation [3]. Simulation methods are often used to design and analyse the effectiveness of automated, guided vehicles in manufacturing plants. Lim et al. used the total travel time, including the waiting and interference out of service breakdown time of vehicles, as the deciding simulation factor for determining the direction of path segments on uni-directional guide path layouts [4]. Um et al. present the simulation design and analysis of a Flexible Manufacturing System (FMS) with an Automated Guided Vehicle system (AGVs). To maximise the operating performance of FMS with AGVs, many parameters were considered, including the number, velocity, and dispatching rule of AGV, part-types, scheduling, and buffer sizes [5]. Vasudevan et al. describe the application of simulation with bottleneck analysis, work measurement, floor space requirements and facility layout analysis, in order to improve the efficiency, and hence the reliability and profitability, of steel-mill manufacturing [6]. Jithavech and Krishnan present a simulationbased method in order to develop an efficient layout design facility, however with uncertainty as to the demand for the product. The validation of the simulation approach, against analytical procedures, was detailed firstly and the methodology for a simulation based approach was then provided. Results from case studies showed that this procedure results in a reduction of risk of as high as 80\% [7]. Yang et al. propose the use of simulation and a digital factory to construct a virtual plant environment in order to implement integration between process planning and manufacturing [8]. Reeb et al. examine how 11 part-families were developed and then selected, using discrete event simulation for cell manufacture and subsequent exclusion from the current manufacturing system of a value-added, wood products company. Using simulation and management input, two of the 11 part-families, representing 51 different part types, were chosen for exclusion from the traditional job shop floor. This exclusion resulted in an average total work-in-process reduction of 112 parts which represented a $17 \%$ reduction [9]. Joseph and Sridharan made an evaluation of the routing flexibility of an FMS with the dynamic arrival of part types for processing in the system. A typical FMS configuration was chosen for detailed study and analysis. The system was set to five different levels of routing flexibility. A discrete-event simulation model was developed to describe the operation of the FMS [10]. Azadeh et al. present a decision-making model for optimisation of operator allocation in a Cellular Manufacturing System (CMS) using the computer simulation method and a genetic algorithm. They determine the number of cross-trained operators and optimum operator layouts with respect to the cellular condition in a CMS using an integrated approach [11].

Hsueh proposes a new design for a bi-directional automated guided vehicle (AGV) system, using simulation, in which two AGVs could exchange their loads, their scheduled transportation tasks and even their vehicle numbers when they move in opposite directions [12].

The problem of maximising the throughput of production lines, by changing buffer sizes, or locations, using simulation methods was studied by Vidalis et al. [13]. The critical literature overview in the area of buffer allocation and production line performance was done by Battini, Persona and Regattieri [14] while Demir, Tunali and Eliiyi proposed a classification scheme to review the studies and presented a comprehensive survey on the buffer allocation problem in production systems [15]. Stanley and Kim presented the results of simulation experiments carried out for buffer allocations in closed, series-production lines [16].

In this article, computer simulation methods are used to analyse the impact of the availability of resources, buffer capacities and the allocation of users in the production system and on throughput and work-in-progress. The research was performed on a 
production system which included 3 manufacturing cells, including 2 CNC machines, and 2 assembly stations. The material flow was directed to the individual machines on the basis of the round robin dispatching rule. The processing time of the machines is determined by uniform distribution, to model random numbers that are located between the interval bounds Start and Stop. In the model of the system presented, production of three various products is simulated. Each product is manufactured using different batch sizes; when changing production batches, set-up times must be taken into consideration.

Generally, the main research problem can be formulated as follows:

Given that a manufacturing system includes machining and assembly operations, and that between individual operations, intermediate buffers are allocated, how, then, do different numbers of workers, buffer capacities and available resources impact on the system's throughput and the average lifespan of the products?

To solve this problem, a set of simulation experiments was conducted in which the input values were the varying capacities of the intermediate buffers, different numbers of workers and variations in the availability of manufacturing resources and where the output values were the throughput of the system and the average lifespan of the products. The motivation of authors is finding general relations among the resources, buffer allocation and effectiveness of the assembly manufacturing system using simulation methods. Solving of the research problem enable to propose algorithms of buffer and resources allocation to obtain determined performance of manufacturing system. The simulation research was performed using Tecnomatix Plant Simulation software. In the next chapter, the simulation model of the production system is described.

\section{A simulation model of a manufacturing system}

The model of the production system was prepared on the basis of an existing example of a manufacturing system, dedicated to the production of metal parts for the automotive sector. The model and simulation experiments were implemented using Tecnomatix Plant Simulation software, version 11.0.0, and are presented in Fig. 1.

The manufacturing system studied included machining operations realised in three production cells, at two CNC machines per cell, and two assembly operations. The machines are marked as ST11, ST12,
ST21, ST22, ST31, ST32 and the assembly stations as AS01 and AS02. Intermediate buffers are allocated between the production cells and the assembly stations. A buffer is also allocated between the two assembly stations. The processing times of the resources are defined on the basis of uniform statistical distribution. Uniform distribution may be used to model a random number that is located between the interval bounds, Start and Stop.

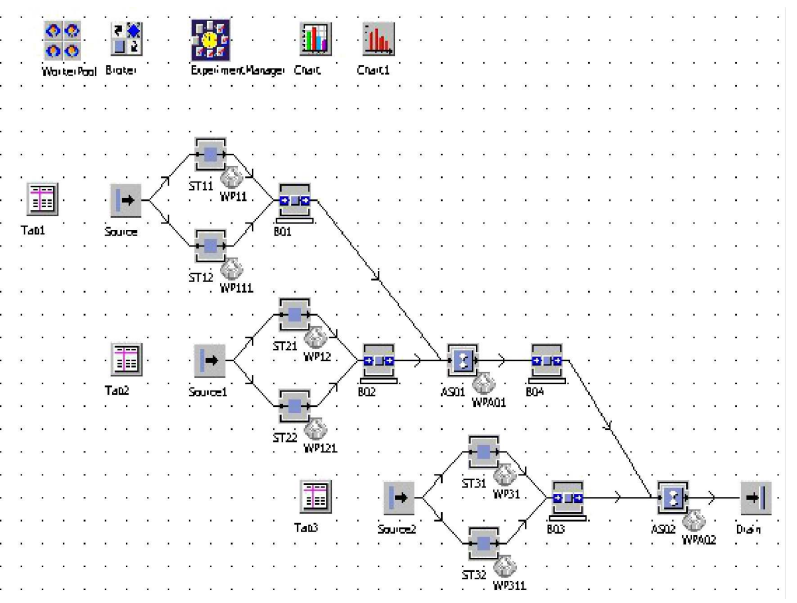

Fig. 1. A simulation model of the manufacturing system.

Uniform distribution can be used when not much is known about the random numbers, that is, the processing times. The probable density function of the uniform distribution for start $<x<$ stop is:

$$
f(x)=\frac{1}{(\text { stop }- \text { start })} .
$$

The mean is:

$$
\mu=\frac{\text { start }+ \text { stop }}{2}
$$

and the variance is

$$
\sigma^{2}=\frac{(\text { stop }- \text { start })^{2}}{2} .
$$

An example of the density function of uniform distribution is presented in Fig. 2.

The processing times in CNC machines is characterised by a discrepancy of about 10 minutes being the difference between Start and Stop. The set-up time for all workstations is determined by uniform distribution where start $=10$ and stop $=20$. The values of processing times for CNC machines are presented in the Table 1.

The results in Table 1 show that assembly operations are much shorter than machining operations. In the system, three batches of products are processed cyclically, where batch sizes are 100, 50 and 300 pieces respectively. In the system, 30 simulation experiments are prepared for the capability 
of different resources and the capacity of the buffers. The experiments are conducted for different numbers of employees. In the next chapter, the results of the simulation experiments are presented.

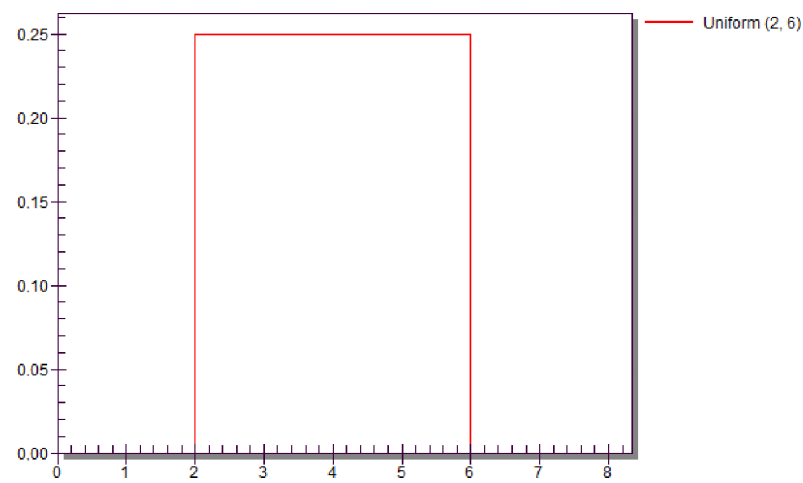

Fig. 2. Density function of uniform distribution for start $=2$ and stop $=6[17]$.

Table 1

Processing times for the production resources.

\begin{tabular}{c|c|c}
\hline Station & Start & Stop \\
\hline ST11 & $5: 00$ & $1: 00$ \\
\hline ST12 & $5: 00$ & $1: 00$ \\
\hline ST21 & $5: 00$ & $1: 00$ \\
\hline ST22 & $5: 00$ & $1: 00$ \\
\hline ST31 & $5: 00$ & $1: 00$ \\
\hline ST32 & $5: 00$ & $1: 00$ \\
\hline AS01 & $1: 00$ & $5: 00$ \\
\hline AS02 & $1: 00$ & $5: 00$ \\
\hline
\end{tabular}

\section{The outcome of computer simulation experiments}

Figure 3 presents the proposed methodology for the simulation research. Research hypotheses are formulated for the activity of the manufacturing system. In the first step of the procedure, the following hypotheses are formulated:

1. An increase in intermediate buffer capacity results in an increase in the throughput of the system.

2. An increase in the availability of manufacturing resources results in an increase in the throughput of the system if buffer capacity is properly allocated.

3. There is an optimal number of workers and buffer capacity allocation which will guarantee maximum throughput of the system.

4. The average lifespan of the products depends on the allocation of buffer capacity, the number of workers and the availability of resources.

The proposed methodology of scientific research include:
- describing of the assembly manufacturing system and execution of a simulated model.

- preparation of the simulation experiments (allocation of the buffer capacity, and resources (CNC machines) availability.

- conducting of the simulation experiments.

- repeat experiments for different numbers of employees.

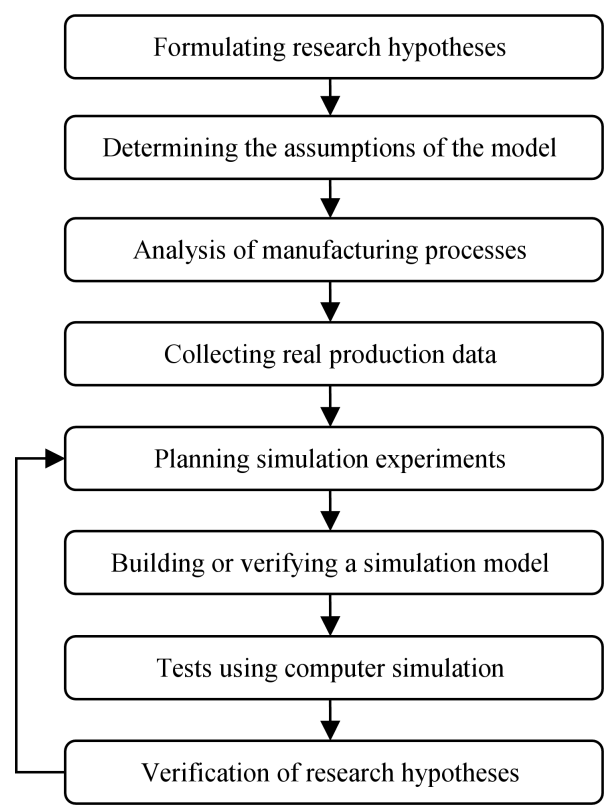

Fig. 3. The procedure for conducting simulation studies

A simulation model is created on the basis of an analysis of an existing manufacturing system; q.v. Fig. 1. Processing and set-up times are defined using statistical distribution selected on the basis of real time measurement. Simulation experiments are carried out in order to verify formulated hypotheses. Input values for the experiments are presented in Table 2.

Variables AS01 and AS02 determines the availability of assembly stations. Variables ST11, ST12, ..., ST32 determines the availability of workstation (CNC machines) and variables B01, B02, .., B04 buffer capacities. Input values for the experiments include the availability of production resources, which changes from $95 \%$ to $99 \%$ and buffer capacity, which changes from 1 to 20 . The combination of the parameters provide 30 experiments. The experiments are conducted for 3,5 and 6 workers. What is interesting is that no additional increase in the number of workers has any impact on improving system throughput.

The first set of experiments is conducted for 3 workers although, as a general rule, there are 8 workplaces in the system. The results of the experiments viz. the throughput and the average product lifespan are presented in Figs. 4a and $4 \mathrm{~b}$ respectively. 
Table 2

Input values defined for experiments.

\begin{tabular}{|c|c|c|c|c|c|c|c|c|c|c|c|c|}
\hline & ST11 & ST12 & ST21 & ST22 & ST31 & ST32 & AS01 & AS02 & B01 & B02 & B03 & B04 \\
\hline Ex01 & 95 & 95 & 95 & 95 & 95 & 95 & 95 & 95 & 1 & 1 & 1 & 1 \\
\hline Ex02 & 96 & 96 & 96 & 96 & 96 & 96 & 96 & 96 & 1 & 1 & 1 & 1 \\
\hline Ex03 & 97 & 97 & 97 & 97 & 97 & 97 & 97 & 97 & 1 & 1 & 1 & 1 \\
\hline Ex04 & 98 & 98 & 98 & 98 & 98 & 98 & 98 & 98 & 1 & 1 & 1 & 1 \\
\hline Ex05 & 99 & 99 & 99 & 99 & 99 & 99 & 99 & 99 & 1 & 1 & 1 & 1 \\
\hline Ex06 & 95 & 95 & 95 & 95 & 95 & 95 & 95 & 95 & 2 & 2 & 2 & 1 \\
\hline Ex07 & 96 & 96 & 96 & 96 & 96 & 96 & 96 & 96 & 2 & 2 & 2 & 2 \\
\hline Ex08 & 97 & 97 & 97 & 97 & 97 & 97 & 97 & 97 & 2 & 2 & 2 & 2 \\
\hline Ex09 & 98 & 98 & 98 & 98 & 98 & 98 & 98 & 98 & 2 & 2 & 2 & 2 \\
\hline Ex10 & 99 & 99 & 99 & 99 & 99 & 99 & 99 & 99 & 2 & 2 & 2 & 2 \\
\hline Ex11 & 95 & 95 & 95 & 95 & 95 & 95 & 95 & 95 & 4 & 4 & 4 & 4 \\
\hline Ex12 & 96 & 96 & 96 & 96 & 96 & 96 & 96 & 96 & 4 & 4 & 4 & 4 \\
\hline Ex13 & 97 & 97 & 97 & 97 & 97 & 97 & 97 & 97 & 4 & 4 & 4 & 4 \\
\hline Ex14 & 98 & 98 & 98 & 98 & 98 & 98 & 98 & 98 & 4 & 4 & 4 & 4 \\
\hline Ex15 & 99 & 99 & 99 & 99 & 99 & 99 & 99 & 99 & 4 & 4 & 4 & 4 \\
\hline Ex16 & 95 & 95 & 95 & 95 & 95 & 95 & 95 & 95 & 5 & 5 & 5 & 5 \\
\hline Ex17 & 96 & 96 & 96 & 96 & 96 & 96 & 96 & 96 & 5 & 5 & 5 & 5 \\
\hline Ex18 & 97 & 97 & 97 & 97 & 97 & 97 & 97 & 97 & 5 & 5 & 5 & 5 \\
\hline Ex19 & 98 & 98 & 98 & 98 & 98 & 98 & 98 & 98 & 5 & 5 & 5 & 5 \\
\hline Ex20 & 99 & 99 & 99 & 99 & 99 & 99 & 99 & 99 & 5 & 5 & 5 & 5 \\
\hline Ex21 & 95 & 95 & 95 & 95 & 95 & 95 & 95 & 95 & 10 & 10 & 10 & 10 \\
\hline Ex22 & 96 & 96 & 96 & 96 & 96 & 96 & 96 & 96 & 10 & 10 & 10 & 10 \\
\hline Ex23 & 97 & 97 & 97 & 97 & 97 & 97 & 97 & 97 & 10 & 10 & 10 & 10 \\
\hline Ex24 & 98 & 98 & 98 & 98 & 98 & 98 & 98 & 98 & 10 & 10 & 10 & 10 \\
\hline Ex25 & 99 & 99 & 99 & 99 & 99 & 99 & 99 & 99 & 10 & 10 & 10 & 10 \\
\hline Ex26 & 95 & 95 & 95 & 95 & 95 & 95 & 95 & 95 & 20 & 20 & 20 & 20 \\
\hline Ex27 & 96 & 96 & 96 & 96 & 96 & 96 & 96 & 96 & 20 & 20 & 20 & 20 \\
\hline Ex28 & 97 & 97 & 97 & 97 & 97 & 97 & 97 & 97 & 20 & 20 & 20 & 20 \\
\hline Ex29 & 98 & 98 & 98 & 98 & 98 & 98 & 98 & 98 & 20 & 20 & 20 & 20 \\
\hline Ex30 & 99 & 99 & 99 & 99 & 99 & 99 & 99 & 99 & 20 & 20 & 20 & 20 \\
\hline
\end{tabular}

a)

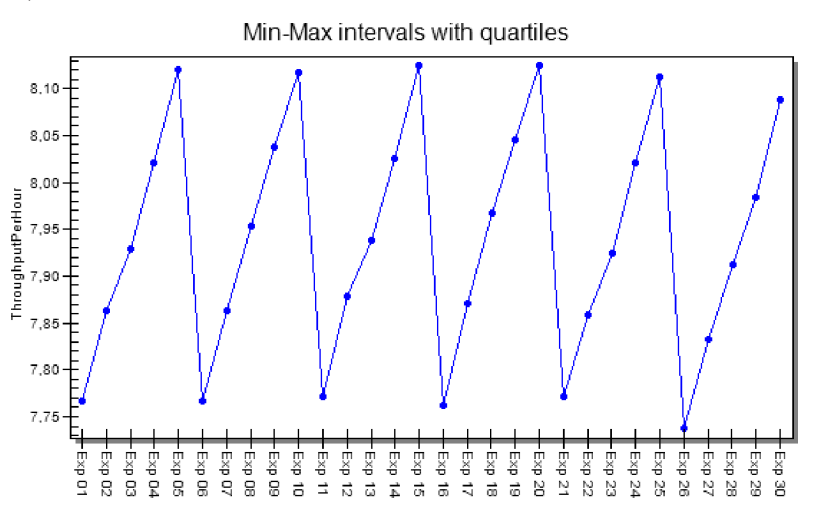

b)

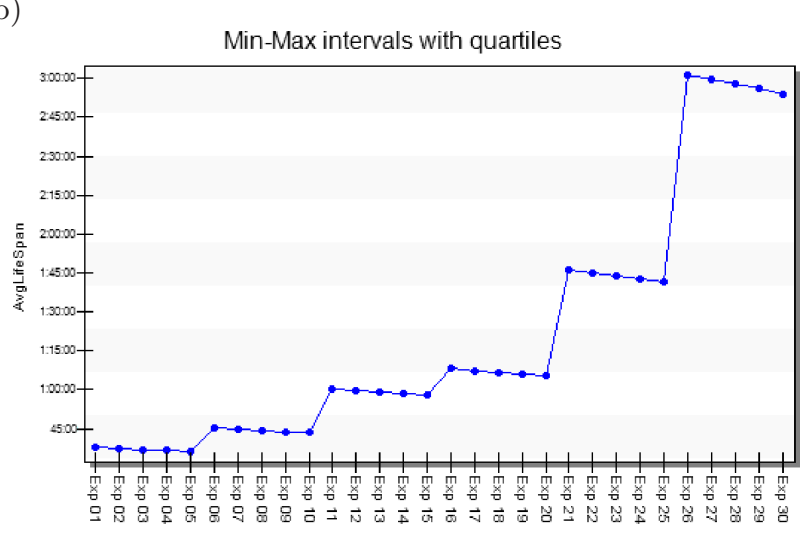

Fig. 4. a) Throughput of the system when serviced by 3 workers, b) average lifespan of products in the system with 3 workers

If the manufacturing system is serviced by 3 workers only, the maximum throughput of the system is reached if the availability of resources is $99 \%$; however, the allocation of buffer capacity does not matter; in fact, the throughput, at maximum buffer capacity, is smaller. 
Maximum throughput of the system equals 8 to 12 products per hour and is achieved with smaller buffer capacities. An increase in buffer capacity impacts on the lifespan of the average product. Fig. 4b shows a time increase in the average residence time of the workpieces in the system from 37 minutes to 2 hours and 53 minutes. The throughput of the system is almost without change.

The resource statistics are presented in Fig. 5. The CNC machines ST11 and St12 are blocked because of the lack of human resources, that is, the lack of staff which blocks the whole system. Generally, resources are used at the rate of $50 \%$. The resources statistics chart, given below, is for the last experiment, that is, $\operatorname{Exp} 30$.

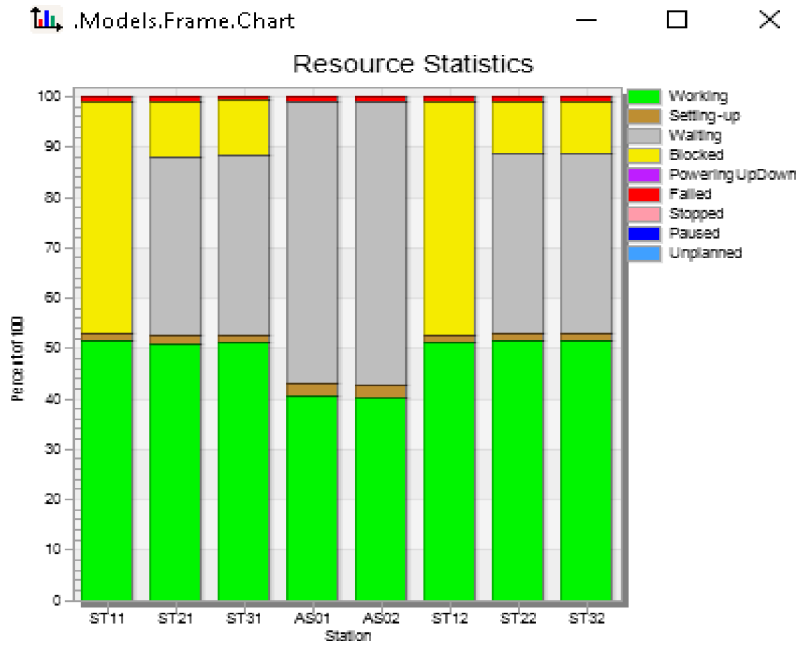

Fig. 5. Resource utilisation for 3 workers.

If the number of workers is increased to 5 , the maximum throughput of the system is increased to 13.55 products per hour. An increase in intermediate buffers has little impact on the throughput; in buffer capacities 1 and 2, the maximum throughput of the system is 13,23 and 13.38 respectively. The values of the throughput are presented in Fig. 6a.

Generally, small buffer capacities result from a small throughput within the system; however for buffer capacities greater than 2, the throughput is solely dependent on the availability of resources.

The average product lifespan (Fig. 6b) is significantly increased where buffer capacities equal 20 and the availability of resources equals $96 \%$, the average lifespan of the product being 1 hour and 24 minutes. Increasing the availability of resources does not always result in any reduction in the average lifespan of the product; - q.v. and compare- the average product lifespan values for experiments 29 and 30 .
The percentage of filled buffers for the last experiment is presented in Fig. 7. The buffers are filled relatively evenly; only buffer B01 is almost filled with 20 products.

a)

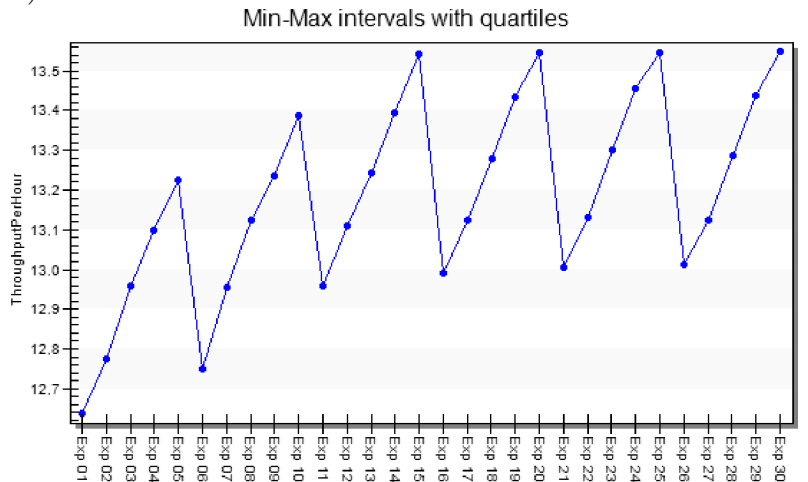

b)

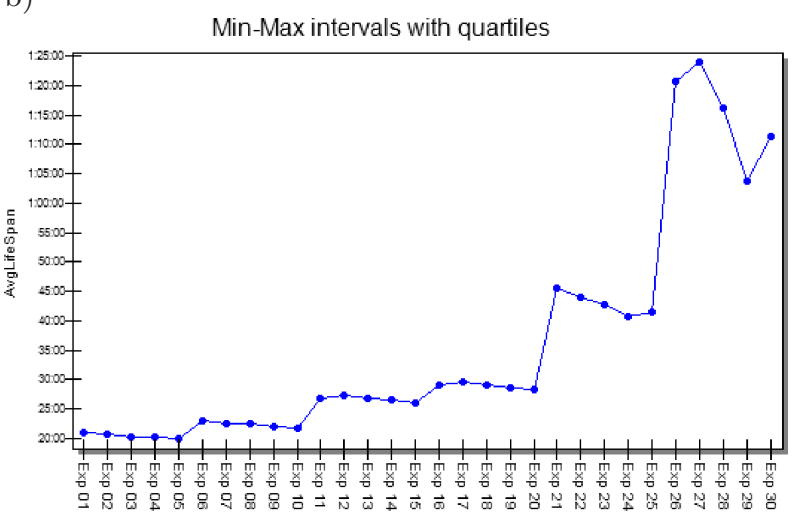

Fig. 6. a) Throughput of the system when serviced by 5 workers, b) average lifespan of products in the system with 5 workers.

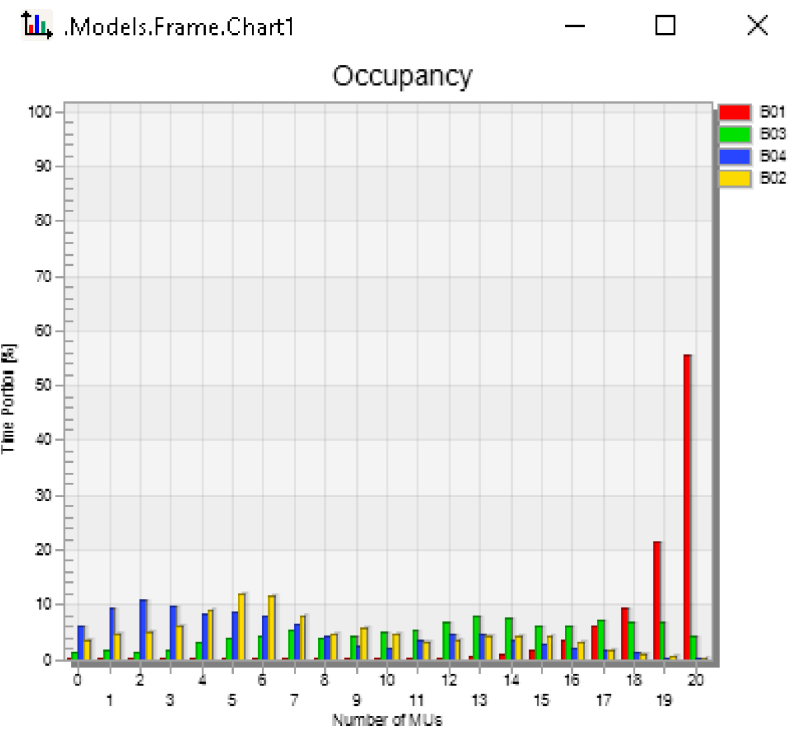

Fig. 7. The buffer occupancy of the system serviced by 5 workers. 
Buffer B04 is almost filled with 3-6 products and buffer B02, with 5 or 6 products. Buffer B03 is usually filled with $16-18$ products. In the next step of the research, the number of workers increases to 6 persons. The results of the throughput and average lifespan are presented in Figs. 8a and 8b. In the system with 6 workers, the impact of the intermediate buffers is significant.

a)

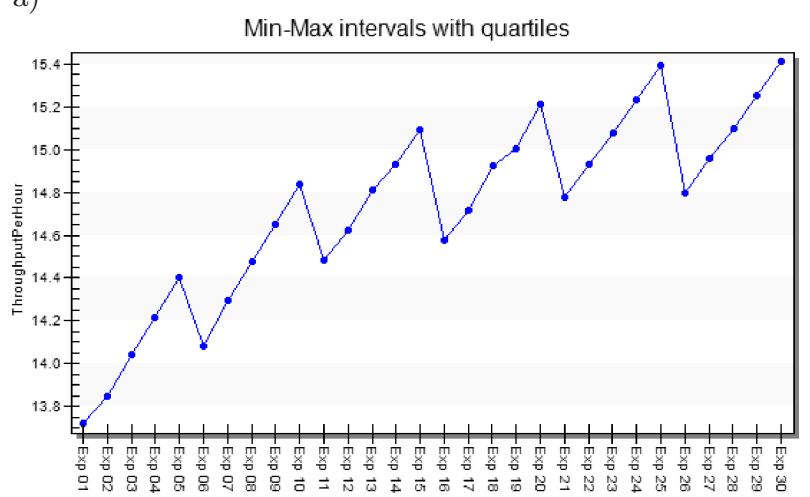

b)

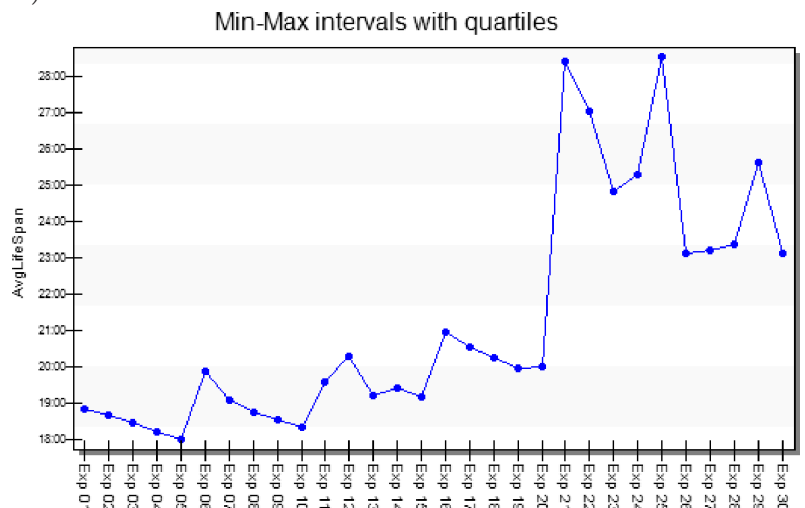

Fig. 8. a) Throughput of the system when serviced by 6 workers, b) average lifespan of products in the system with 6 workers.

The best value of the throughput is reached with buffer capacities of 10 and 20 which are practically the same values as for the analogical values of the availability of resources. As can be seen from the chart presented in fig. 8a, the results obtained for the various combinations of the availability of resources and buffer capacities, indicate that the same level of throughput can be reached. For example, in the system with an availability of resources of $99 \%$ and buffer capacities of 2 , the same throughput can be reached as for the system with an availability of resources of $96 \%$ and buffer capacities of 4 , with their respective throughputs being 14,65 and 14,62 products per hour. The average lifespan of products for the system with 6 workers is presented in Fig. 8b.
The significantly shorter stay of products within the system results in a smaller number of works-inprogress.

The greatest value for the average lifespan of products, that is, more than 28 minutes, is reached with a buffer capacity of 10 and a resources availability of $95 \%$ and $99 \%$. For the final experiments, all resources without assembly stations are utilised maximally, see Fig. 9, which means that by using 6 workers, the system obtains maximum effectiveness of production. The occupancy chart for intermediate buffers for 6 workers and Exp 30 is presented in Fig. 10.

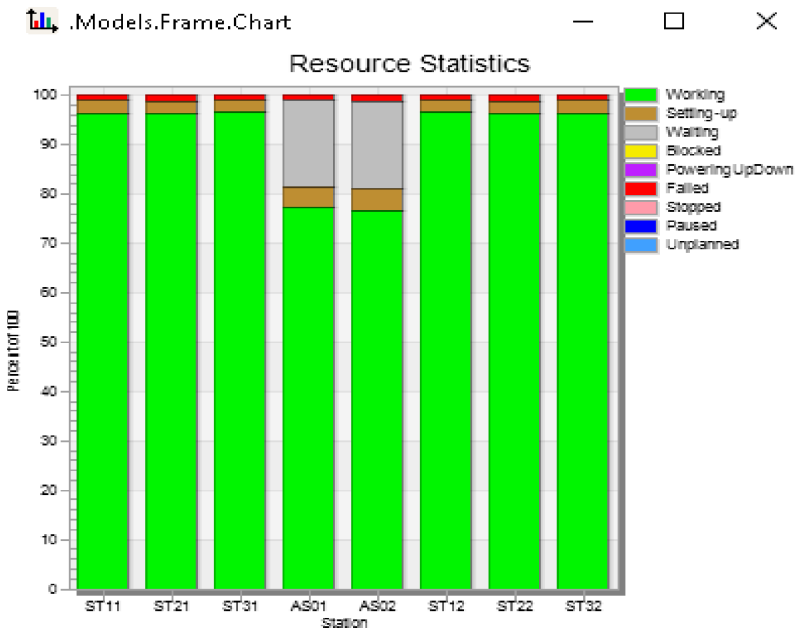

Fig. 9. Resource utilisation for 6 workers.
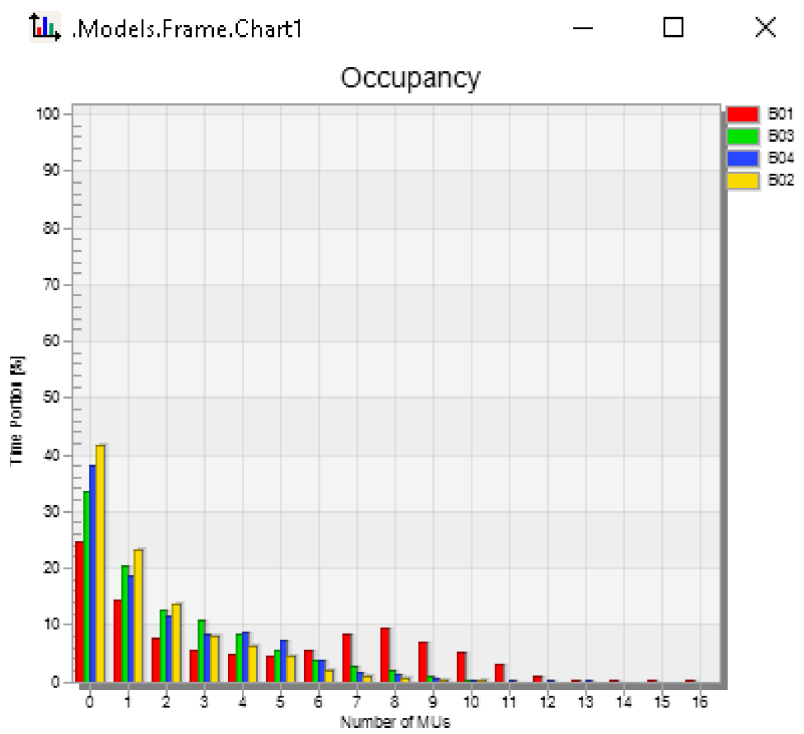

Fig. 10. Buffer occupancy of the system serviced by 6 workers.

Buffers primarily hold from 0 to 5 products with only buffer B01 including more products (0-10). Further increases in the number of workers has no im- 
Management and Production Engineering Review

pact on the throughput of the system or on the average lifespan of the products. From the analysis of the experiments, it can be seen that the maximum throughput of the production system, viz. more than 15 products per hour, can be obtained with the maximum availability of resources and with the maximum number of users and with an intermediate buffer capacity greater than 10 .

Increasing the capacity of intermediate buffers, however, results in an increase in work-in-progress, that is, in the average lifespan of the products. The greater the availability of manufacturing resources, then the greater is the requirement to use new CNC machinery and assembly stations.

To evaluate the objective impact of the selected parameters on the efficiency of the system, a new efficiency index $\gamma$ is proposed, $q . v$.

$$
\gamma=\frac{\mathrm{E}}{\alpha \cdot \varphi \cdot v} \cdot 100
$$

where $\mathrm{E}$ - throughput of the system, $\alpha$ - average resources availability, $\varphi-$ number of workers, $v-$ average lifespan of products.

The efficiency index $\gamma$ is proposed to present in simple form, algebraic relation among throughput, number of workers, resources availability and lifespan (buffer capacity).

The index can be used to evaluate generally behavior of the system. If some parameters are more important, weight values can be used. The values of the efficiency index for experiments are presented in Table 3 and Fig. 11.

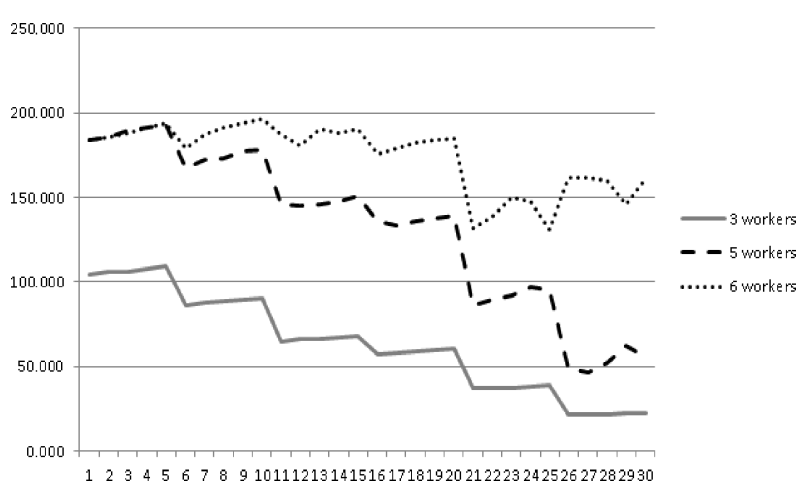

Fig. 11. Values for efficiency index $\gamma$ for the experiments.

In the table, efficiency indices greater than 190 are marked. The greatest values are obtained for experiment 05 for 5 and 6 workers and experiments 09 and 10 for 6 workers.

Generally, increasing intermediate buffer capacity results in an increase in the average lifespan of products or work-in-progress. In the next chapter, the final conclusions and directions for further research are presented.
Table 3

Resource utilisation for 6 workers.

\begin{tabular}{|c|c|c|c|}
\hline & 3 workers & 5 workers & 6 workers \\
\hline Exp 01 & 104,358 & 183,817 & 184,145 \\
\hline Exp 02 & 105,765 & 185,864 & 185,505 \\
\hline Exp 03 & 106,316 & 189,477 & 188,477 \\
\hline Exp 04 & 107,661 & 191,354 & 191,375 \\
\hline Exp 05 & 109,269 & 193,368 & 194,188 \\
\hline Exp 06 & 86,644 & 167,375 & 178,999 \\
\hline Exp 07 & 87,880 & 172,184 & 187,449 \\
\hline Exp 08 & 89,028 & 173,493 & 191,297 \\
\hline Exp 09 & 89,887 & 177,506 & 193,765 \\
\hline Exp 10 & 90,742 & 178,549 & 196,228 \\
\hline Exp 11 & 64,898 & 146,228 & 187,075 \\
\hline Exp 12 & 65,990 & 144,795 & 180,399 \\
\hline Exp 13 & 66,317 & 146,184 & 190,881 \\
\hline Exp 14 & 66,964 & 147,721 & 188,518 \\
\hline Exp 15 & 67,925 & 150,959 & 190,897 \\
\hline Exp 16 & 57,472 & 135,689 & 175,974 \\
\hline Exp 17 & 58,466 & 133,357 & 179,036 \\
\hline Exp 18 & 59,250 & 135,917 & 182,585 \\
\hline Exp 19 & 59,732 & 137,831 & 184,200 \\
\hline Exp 20 & 60,388 & 139,000 & 184,569 \\
\hline Exp 21 & 37,015 & 86,495 & 131,388 \\
\hline Exp 22 & 37,456 & 89,441 & 138,119 \\
\hline Exp 23 & 37,719 & 92,406 & 150,376 \\
\hline Exp 24 & 38,236 & 97,327 & 147,501 \\
\hline Exp 25 & 38,680 & 94,959 & 130,755 \\
\hline Exp 26 & 21,550 & 48,881 & 161,699 \\
\hline Exp 27 & 21,812 & 46,835 & 161,263 \\
\hline Exp 28 & 22,010 & 51,862 & 159,994 \\
\hline Exp 29 & 22,208 & 61,882 & 145,838 \\
\hline Exp 30 & 22,537 & 55,178 & 161,736 \\
\hline
\end{tabular}

\section{Conclusions}

In this paper, an analysis of the throughput and life-span of a manufacturing system, using computer simulation, is presented. Simulation experiments were conducted for different capacities of intermediate buffers, different numbers of workers and for the different availability of manufacturing resources $[18$, 19]. A model of a manufacturing system was prepared using Tecnomatix Plant Simulation software on the basis of an actual manufacturing system [20]. The processing times were defined using uniform, statistical distribution. Three sets of experiments were prepared for 3,5 and 6 workers. The research hypotheses are formulated and confirmed by the simulation research.

To evaluate the research experiments, an efficiency system was defined. The index enables the rela- 
tionship between the throughput, the availability of resources, the number of workers and the average lifetime of the products to be analysed. Using the index from all the experiments and the allocation of intermediate buffers, the availability of resources within the system is selected which guarantees the optimum efficiency of the system.

The results of the simulation research has facilitated the formulation of the following conclusions for the model of the manufacturing system investigated:

- the capacity of intermediate buffers can significantly impact on the throughput of the manufacturing system,

- to evaluate the general efficiency of the throughput of a manufacturing system, the-work-inprogress, the number of workers and the availability of resources should all be taken into account,

- to obtain maximum throughput within a system, one worker should be allocated to a specific manufacturing resource, that is, 8 resources with only 6 workers,

- the same system throughput can be obtained for different combinations of the availability of resources, buffer capacities and numbers of workers.

The results presented in the paper could be implemented in industrial practice by allocation of personnel for assembly manufacturing system and allocation of buffer capacity when the availability of manufacturing resources are known. Further research will focus on finding a formal relationship between the throughput of the system, the average lifespan of the products, the buffer capacities, the number of workers and the availability of resources.

\section{References}

[1] Negahban A., Smith J.S., Simulation for manufacturing system design and operation: literature review and analysis, Journal of Manufacturing Systems, 33, 241-261, 2014.

[2] Jahangirian M., Eldabi T., Naseer A., Stergioulas L.K., Young T., Simulation in manufacturing and business: a review, European Journal of Operational Research, 203, 1, 1-13, 2010.

[3] Jagstam M., Klingstam P., A handbook for integrating discrete event simulation as an aid in conceptual design of manufacturing systems, Proceedings of the 2002 Winter Simulation Conference, 2, 1940-4, 2002 .

[4] Lim J.K., Lim J.M., Yoshimoto K., Kim K.H., Takahashi T., A construction algorithm for designing guide paths for automated guided vehicle systems, International Journal of Production Research, 40, 15, 3981-94, 2002.
[5] Um I., Cheon H., Lee H., The simulation design and analysis of a flexible manufacturing system with automated guided vehicle system, Journal of Manufacturing Systems, 28, 4, 115-22, 2009.

[6] Vasudevan K., Lammers E., Williams E., Ulgen O., Application of simulation to the design and operation of steel mills devoted to the manufacture of pipelines, Second International Conference on Advances in System Simulation (SIMUL), pp. 1-6, 2010 .

[7] Jithavech I., Krishnan K., A simulation-based approach to the risk assessment of facility layout designs under stochastic product demands, The International Journal of Advanced Manufacturing Technology, 49, 27-40, 2010.

[8] Yang T., Zhang D., Chen B., Li S., Research on plant layout and production line running simulation in digital factory environment, Pacific-Asia Workshop on Computational Intelligence and Industrial Application, 2, 588-593, 2008.

[9] Reeb J., Baker E., Brunner C., Funk J., Reiter W., Using simulation to select part-families for cell manufacturing, International Wood Products Journal, 1, 1, 43-7, 2010.

[10] Joseph O.A., Sridharan R., Simulation modelling and analysis of routing flexibility of a flexible manufacturing system, International Journal of Industrial and Systems Engineering, 8, 1, 61-82, 2011.

[11] Azadeh A., Anvari M., Ziaei B., Sadeghi K., An integrated fuzzy DEA-fuzzy-means-simulation for optimisation of operator allocation in cellular manufacturing systems, The International Journal of Advanced Manufacturing Technology, 46, 1-4, 361-75, 2010.

[12] Hsueh C.F., A simulation study of a bi-directional load-exchangeable automated guided vehicle system, Computers \& Industrial Engineering, 58, 4, 594601,2010

[13] Vidalis M.I., Papadopoulos C.T., Heavey C., On the workload and 'phase load' allocation problems of short reliable production lines with finite buffers, Computers and Industrial Engineering, 48, 825-837, 2005.

[14] Battini D., Persona A., Regattieri A., Buffer size design linked to reliability performance: a simulative study, Computers \& Industrial Engineering, 56, 1633-1641, 2009.

[15] Demir L., Tunali S., Eliiyi D.T., The state of the art on the buffer allocation problem: a comprehensive survey, Journal of Intelligent Manufacturing, 25, 371-392, 2014. 
[16] Staley D.R., Kim D.S., Experimental results for the allocation of buffers in closed serial production lines, International Journal of Production Economics, 137, 284-291, 2012.

[17] Tecnomatix Plant Simulation 11 - on-line documentation.

[18] Kłos S., Patalas-Maliszewska J., Trebuňa P., Improving manufacturing processes using simulation methods, Applied Computer Science, 12, 4, 7-17, 2016.
[19] Kłos S., Trebuňa P., Using computer simulation method to improve throughput of production systems by buffers and workers allocation, Management and Production Engineering Review, 6, 4, 60-69, 2015.

[20] Kłos S., The simulation of manufacturing systems with tecnomatix plant simulation, Zielona Góra, Oficyna Wydaw. Uniwersytetu Zielonogórskiego, pp. $124,2017$. 